\title{
Power and Temperature Prediction for Computer System Power Optimization
}

\author{
Koji Nishi $^{1} \quad$ Shota Takada $^{1} \quad$ Takamichi Kaneda $^{1}$ \\ ${ }^{1}$ Department of Innovative Engineering, Ashikaga University, Japan, nishi.koji@v90.ashitech.ac.jp
}

\begin{abstract}
This paper investigates desktop computer system power optimization with Modelica. Firstly, microprocessor power equation is modeled with voltage and temperature dependency. Secondly, steady state heat transfer path is modeled as a variable thermal resistance with fan power change and is connected to microprocessor power model to discuss optimum working point of fan which minimizes power consumption of the power rail for the microprocessor on the target computer system under CPU-intensive workload condition. After that, transient heat transfer path modeling is explored as a Cauer thermal network creation.
\end{abstract}

Keywords: Computer system, Microprocessor, Power Optimization

\section{Introduction}

Computer systems are vital in the modern world. Higher performance is still explored not only for high performance computing systems and server systems but also for consumer computer systems such as desktop computers, notebook computers and slate devices. Also, IoT (Internet of Things) and M2M (Machine-toMachine) infrastructure has been rapidly developed in a few years and computer systems for these use will be increased rapidly. While, energy consumption needs to be eliminated in the sustainable development of modern society and power consumption by computer systems is one of important subject to be solved.

The latest microprocessors incorporate power management features which control trade-off of computing performance and power consumption. With these features, microprocessor's power efficiency has been improved drastically in the past decade, however, system level power optimization is still on the way because system level power optimization needs to consider power consumption of not only microprocessor itself but also peripherals and power supply circuit for them. And power consumption of the microprocessor is highly dependent on temperature, however, current system-level temperature control methodology is to maintain junction temperature of microprocessor within operational temperature range and not to minimize system-level power consumption.

To minimize system-level power consumption, power modeling methodology with practical accuracy is required. There are many efforts and researches on electrical characteristics on CMOS (Complementary Metal-Oxide-Semiconductor) circuits. And microprocessors incorporate power management features, while system-level power optimization is still on the way. Thus, author investigates microprocessor power equation to conduct both power and thermal control as a part of previous study (Nishi, 2012). In the study, microprocessor power equation with voltage and temperature dependencies is introduced and is employed in three-dimensional heat conduction simulation. However, three-dimensional simulation costs too much from a computational load standpoint and takes time to create accurate thermal simulation model. To enable fast simulation environment, threedimensional simulation needs to be replaced by another methodology which ensures high speed calculation with practical accuracy. Also, model creation effort should be minimized as much as possible.

As another methodology to conduct thermal simulation with reduced computational load, there are thermal network methodology and model order reduction (MOR). Thermal network methodology has long history for temperature prediction. As for thermal network application to semiconductor devices, there already exist some researches in 1980s. For example, thermal network is utilized for temperature prediction of multi-chip module (Ishizuka and Fukuoka, 1986). Traditional way to create thermal network is to determine thermal resistance and heat capacitance values from dimensions and thermophysical properties of each component along heat transfer paths. In recent years, curve fitting technique to determine thermal resistance and heat capacitance values from measurement data is also sometimes employed. On the other hand, MOR is a technique to reduce computational complexity of simulation model and is sometimes utilized with Modelica. For example, MOR is applied to FEM (Finite Element Method) model and reduced order model is implemented as FMU (Functional Mockup Unit) (Gödecke et al, 2012). Also, MOR based technique is proposed for thermal simulation as well (Codecasa et al, 2014). MOR is useful if threedimensional simulation model or dimensions and 
thermophysical properties of each component along heat transfer paths are available.

This research aims to establish fast simulation environment for computer system power optimization with Modelica. In this paper, power equation is modeled as a Modelica component. Secondly, steady state heat transfer path is modeled as a Modelica component with a variable thermal resistance which expresses fan speed change of heat sink fan over the microprocessor. Thirdly, they are connected each other, and system-level power optimization is investigated. After that, transient heat transfer path modeling is explored as a Cauer thermal network creation, to establish transient control optimization environment.

\section{Target Desktop Computer System}

\subsection{Hardware Configuration}

The target computer system in this paper is a desktop personal computer, Sycom Radiant GZ2650X470A (Fig. 1 (a)). It employs a AMD Ryzen $51500 \mathrm{X}$ processor, which is fabricated with $14 \mathrm{~nm}$ process technology and has $4 \mathrm{CPU}$ cores with totally 8-thread execution capability. Its thermal design power (TDP) is $65 \mathrm{~W}$. The base frequency of the microprocessor is $3.5 \mathrm{GHz}$, however, the microprocessor supports boosted frequency up to $3.7 \mathrm{GHz}$. Under lower temperature conditions, it runs over $3.5 \mathrm{GHz}$ with higher power consumption than TDP. Thus, a large heat sink fan prepared by microprocessor manufacturer is attached over the microprocessor package (Fig. 1 (b)). Table 1 shows major hardware components of the target computer system. The microprocessor consumes more than half of the computer system's power under CPUintensive workload scenario and this paper focuses on microprocessor power.

\subsection{Key Software Components}

Key software components of the target computer system are listed in Table 2. The system is based on Ubuntu

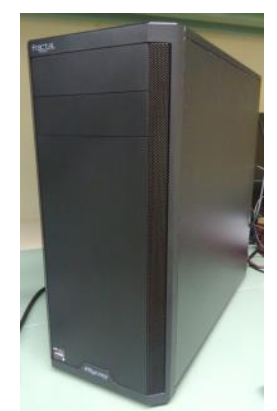

(a) System outlook

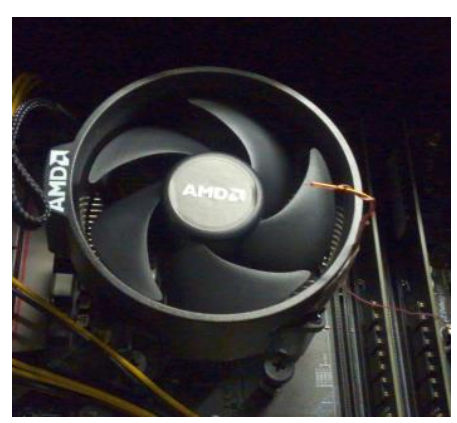

(b) Heat sink fan
Figure 1. Desktop personal computer outlook and heat sink fan for microprocessor.
16.04 (64-bit). Prime 95 is an application software and is utilized as a CPU-intensive workload in this paper. It processes FFT (Fast Fourier Transformation) continuously. Prime 95 has some test modes and "small FFT" is utilized in this paper. AMD $\mu$ Prof is utilized as a monitoring software tool which can log CPU temperature, estimated power consumption and so on.

\section{Power Modeling}

\subsection{Microprocessor Power Equation}

\subsubsection{Power Equation of CMOS digital circuits}

Power consumption of CMOS digital circuits, including the latest microprocessor, is due to electrical charge and discharge to/from load capacitance $C_{\text {load }}$ and power loss by leakage current (Hiramoto et al, 2009). Therefore, its power consumption can be modeled as the equation below (Kunimine et al, 2011):

$$
P_{C M O S}=a C_{\text {load }} V_{D D}^{2} f_{\text {op }}+I_{\text {leak }} V_{D D}
$$

Here, $a$ is activation rate, $C_{\text {load }}$ is load capacitance, $V_{D D}$ is power supply voltage, $f_{o p}$ is operational frequency and $I_{\text {leak }}$ is leakage current. Unfortunately, it is known that $C_{\text {load }}$ has voltage dependency (Uchida et al, 2001; Uchida et al, 2002; Watanabe et al, 2007). Also, there are several types of leakage current (Hiramoto et al,

Table 1. Major hardware components of target desktop computer system.

\begin{tabular}{|l|l|}
\hline Function & Component \\
\hline Microprocessor & $\begin{array}{l}\text { AMD } \\
\text { Ryzen 5 1500X }(65 \mathrm{~W} \text { TDP })\end{array}$ \\
\hline Motherboard & $\begin{array}{l}\text { MSI } \\
\text { B350 TOMAHAWK }\end{array}$ \\
\hline Memory & DDR4-2400 $(16 \mathrm{~GB} \times 2)$ \\
\hline Graphics card & $\begin{array}{l}\text { NVIDIA } \\
\text { GeForce GT710 }(1 \mathrm{~GB})\end{array}$ \\
\hline HDD & $\begin{array}{l}\text { Toshiba } \\
\text { DT01ACA100 }(1 \mathrm{~TB})\end{array}$ \\
\hline Power supply & $\begin{array}{l}\text { Antec } \\
\text { NeoECO NE650C }(650 \mathrm{~W})\end{array}$ \\
\hline
\end{tabular}

Table 2. Major software components of target desktop computer system.

\begin{tabular}{|l|l|}
\hline Function & Component \\
\hline Operating system & Ubuntu 16.04 (64-bit) \\
\hline $\begin{array}{l}\text { Software utilized as } \\
\text { CPU workload }\end{array}$ & $\begin{array}{l}\text { Prime 95 v29.5 } \\
\text { (Linux version 64-bit) }\end{array}$ \\
\hline $\begin{array}{l}\text { Processor information } \\
\text { gathering }\end{array}$ & $\begin{array}{l}\text { AMD } \mu \text { Prof v1.2.275.0 } \\
\text { (Linux version 64-bit) }\end{array}$ \\
\hline
\end{tabular}


2009). Many of them have voltage dependency. Moreover, subthreshold current, which is a dominant factor of leakage current has not only voltage dependency but also temperature dependency.

\subsubsection{CPU Core Power Equation}

For microprocessor CPU cores, $V_{D D}$ needs to be changed when $f_{o p}$ is changed to increase computational performance or lower power consumption. Thus, $C_{\text {load }}$ is changed when $f_{o p}$ is changed. Author investigated the way to express $C_{\text {load }}$ for microprocessor silicon die temperature prediction and concluded linear approximation of $V_{D D}$ works well because $V_{D D}$ range of CPU core is not so much large during normal operation as a part of previous study (Nishi, 2012). As for leakage current, subthreshold current is modeled as exponential function of temperature for precise discussion (Amelifard, 2008). However, exponential function is not suitable for high speed electro-thermal simulation, especially for transient simulation, from a computational load standpoint. Also, microprocessor is utilized under temperature range from room ambient to around $100 \mathrm{deg} \mathrm{C}$ and it is relatively small range compared to other semiconductor devices such as power semiconductor. Thus, Author investigated the way to express leakage current for microprocessor silicon die temperature prediction and concluded linear approximation of $V_{D D}$ and quadratic approximation of silicon die temperature $T$ work well as a part of previous study (Nishi, 2012). Therefore, power consumption of a CPU core can be expressed as the equation below:

$$
\begin{aligned}
P_{C P U}= & \left(d_{1} V_{D D}+d_{2}\right) V_{D D}^{2} f_{O p} \\
& +\left(s_{1} V_{D D}+s_{2}\right)\left(T^{2}+s_{3} T+s_{4}\right) V_{D D}
\end{aligned}
$$

Here, $d_{1}, d_{2}$ are coefficients of the first term, called as dynamic power term, and $s_{1}$ to $s_{4}$ are coefficients of the second term, called as static power term, on the right in eq. (2).

\subsubsection{Microprocessor Power Equation}

Microprocessor has several power rails and sum of their power consumption equals to microprocessor power consumption. Since this paper focuses on CPU intensive workload case only and microprocessor power consumption other than CPU cores is relatively small and can be modeled as constant. Therefore, microprocessor power consumption in the case that all CPU cores run the same workload is

$$
P_{\text {Microprocessor }}=n P_{C P U}+P_{\text {Other }}
$$

Here, $n$ is number of CPU cores and $P_{O t h e r}$ is power comsumption by circuits other than CPU cores in microprocessor silicon die. Applying junction temperature $T_{J}$, which is reported as Die SoC temperature in AMD $\mu$ Prof, as silicon die temperature for $P_{C P U}$ and assuming $P_{\text {other }}$ is constant, the microprocessor power equation is obained finally from eq. (2) and (3),

$$
\begin{aligned}
& P_{\text {Microprocessor }}=\left(c_{1} V_{D D}+c_{2}\right) V_{D D}^{2} f_{o p} \\
& \quad+\left(c_{3} V_{D D}+c_{4}\right)\left(T_{J}^{2}+c_{5} T_{J}+c_{6}\right) V_{D D}+c_{7}
\end{aligned}
$$

Here, $c_{1}$ to $c_{7}$ are coefficients of microprocessor power consumption. By determining these coefficients, microprocessor power consumption can be calculated.

\subsection{Power Consumption of Microprocessor Power Rail}

On the latest desktop computer system, EPS12V power rail works as microprocessor dedicated power supply rail from power supply unit to motherboard (Fig. 2). Since EPS12V has power loss by voltage regulators which convert $12 \mathrm{~V}$ voltage level to several lower voltage levels for the microprocessor, EPS12V is thought as the key power rail for the target desktop computer system under CPU-intensive workload. Thus, not only microprocessor power but also EPS12V power are collected and modeled in this paper. Since EPS12V power is sum of microprocessor power and power loss of DC-DC conversion by voltage regulators of microprocessor power rails, it should take the same form as eq. (4) with different coefficient values from microprocessor.

\subsection{Power Modeling of The Target Desktop Computer System}

Microprocessor and EPS12V power are sensitive to application workload and usual application software changes activation rate in eq. (1) and eq. (2) dynamically. To collect consistent and accurate voltage and

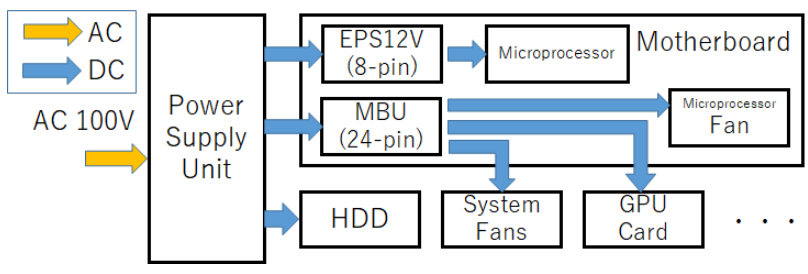

Figure 2. Power supply structure of target desktop computer system.

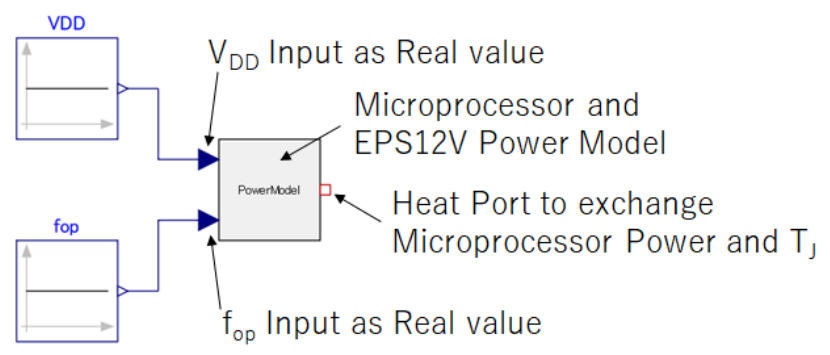

Figure 3. Microprocessor and EPS12V power model with $V_{D D}$ and $f_{o p}$ inputs. 
temperature dependent power data, constant workload should be added. In this paper, M172031, one of small FFT execution in "Torture Test" of Prime 95, is utilized and is added to all CPU threads. Totally eight M172031 modules run on target desktop computer system during mesurement.

AMD $\mu$ Prof tool is utilized to read junction temperature and estimated microprocessor power. EPS12V power rail current and voltage are measured by current probe and data logger, respectively.

OpenModelica v1.13.2 is utilized to create Modelica models and conduct simulation in this paper. Figure 3 shows a created Modelica component model which calculates microprocessor and EPS12V power and $T_{J}$. Coefficients are obtained as parameters and only $V_{D D}$ and $f_{o p}$ are externally obtained via connectors. A heat port is prepared to exchange microprocessor power and $T_{J}$ with external component. Two Eq. (4), one for microprocessor and another for EPS12V, are expressed as Modelica equations. Figure 4 shows Modelica simulation result in the case that an ambient temperature component is connected to heat port of the power model and ambient temperature value is sweeped from $40 \mathrm{deg}$ $\mathrm{C}$ to $70 \mathrm{deg} \mathrm{C}$.

\section{Heat Transfer Path Modeling}

To obtain power consumption under real environment, junction temperature also needs to be calculated. Thus, heat transfer path is modeled as the thermal resistance from junction to ambient, $R_{t h}, J A$, which can be utilized in steady state simulation (Nishi et al, 2019).

$$
R_{\text {th,JA }}=\frac{T_{J}-T_{A}}{P_{\text {Microprocessor }}}
$$

Here, $T_{A}$ is ambient temperature.

Measurement is conducted with the same condition described in section 3.3 and thermal resistance is modeled as a function of fan power.

Figure 5 shows a created Modelica component model which calculates $R_{t h, J A}$ from fan power input value. At this time, $R_{t h, J A}$ is modeled as polynomial which is obtained by curve fitting of measurement result. Figure 6 shows thermal resistance variation by fan power.

\section{Power Optimization Under Steady State}

To obtain both microprocessor power and junction temperature, the equation below needs to be solved:

$$
T_{J}=R_{\text {th,JA}} \cdot P_{\text {Microprocessor }}+T_{A}
$$

In Modelica, eq. (6) is solved by connecting created Modelica component models in Fig. 3 and Fig. 5 as shown in Fig. 7.

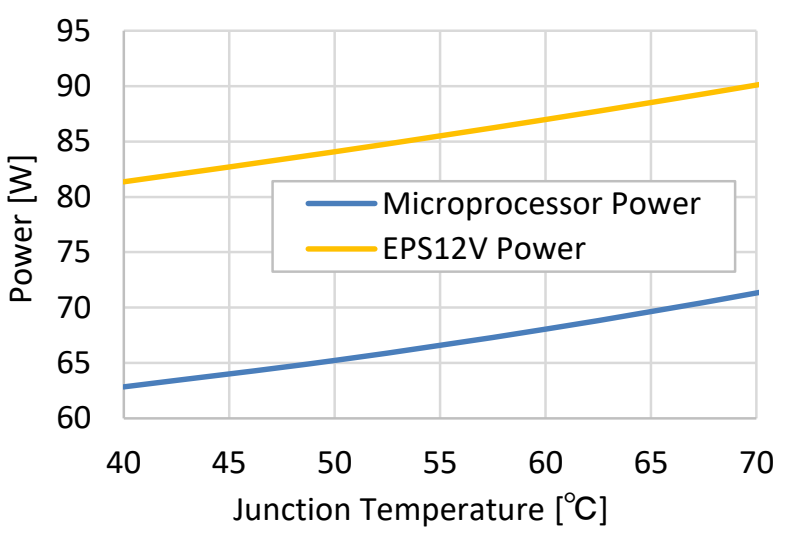

Figure 4. Relationship between junction temperature and power consumption.

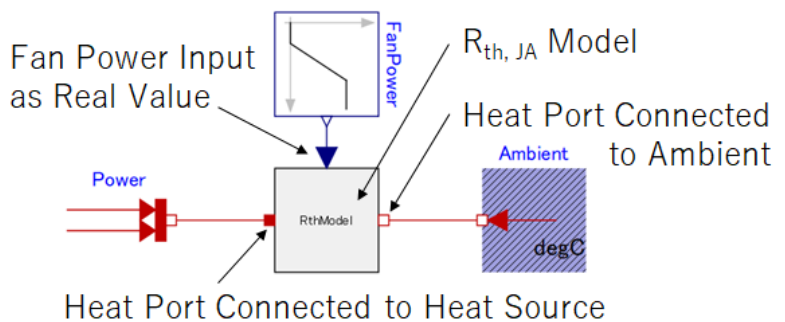

Figure 5. Thermal resistance model with fan power input and connections with heat source and ambient.

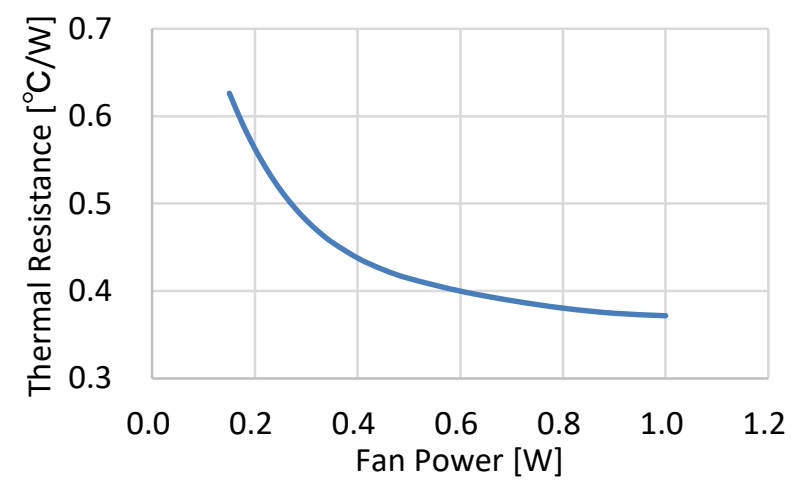

Figure 6. Relationship between fan power and thermal resistance from junction to ambient.

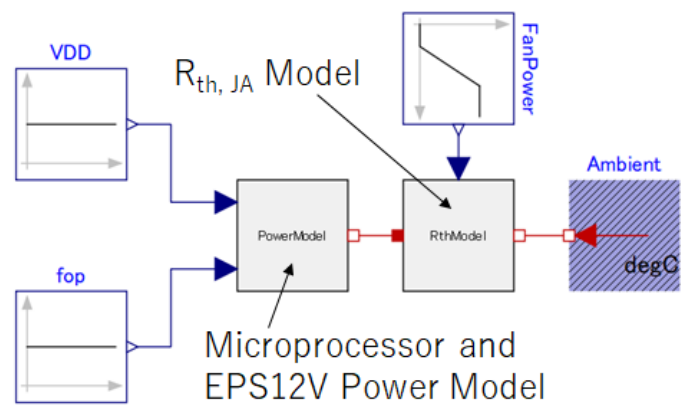

Figure 7. Modelica model connection to calculate both power and junction temperature. 
Figure 8 shows junction temperature variation by fan power, changing ambient temperature. As fan power becomes higher, junction temperature becomes lower, which results in microprocessor power decrease. Figure 9 shows relationship between power consumption, junction temperature and fan power under $30 \mathrm{deg} C$ ambient as an example. Not only microprocessor power but also EPS12V power decrease monotonically by fan power increase.

EPS12V power is dominant and occupies more than half of total power which PSU (Power Supply Unit) provides. To consider system-level power optimization, not only EPS $12 \mathrm{~V}$ power but also fan power should be taken into account. Thus, sum of EPS12V power and fan power (hereafter, target power) is checked as shown in Fig. 10. Fan power is relatively small, however, target power has the minimum value. Figure 11 shows derivative of target power. Target power becomes minimum when its derivative becomes zero. Optimum fan power which shows minimum target power is $\sim 0.84$ $\mathrm{W}$ at $T_{A}=10 \operatorname{deg} \mathrm{C}, \sim 0.93 \mathrm{~W}$ at $T_{A}=50 \operatorname{deg} \mathrm{C}$, respectively. This means an optimum working point moves by ambient condition. Since application workload with usual application software varies time by time, fan needs to be controlled time by time. Optimum working point under lower workload scenario can be obtained by applying the same methodology.

\section{Transient Simulation}

Under steady state condition, optimum fan power is obtained easily with a few Modelica components. Instead, fan control simulation is required to obtain optimum target power under transient state condition because temperature doesn't change immediately and temperature variation is delayed by the effect of heat capacitance along heat transfer paths. Thus, heat transfer path modeling should be carefully for transient simulation and is discussed in this chapter.

Transient heat transfer path can be obtained by extending thermal resistance to RC network. There are two RC network topologies; Cauer and Foster. Cauer $\mathrm{RC}$ thermal network is employed in this paper (Fig. 12). To determine $R_{t h}$ and $C_{t h}$ values, measurement is conducted in the case that microprocessor starts to execute eight M172031 modules from idle state on the target computer system and fitting is executed to minimize the equation:

$$
\begin{aligned}
& \text { eval } \\
& =\sqrt{\frac{1}{t} \int_{0}^{t}\left(T_{J, \text { measured }}-T_{J, \text { estimated }}\right)^{2} d t}
\end{aligned}
$$

Here, $t$ is the total execution time (250 sec), $T_{J \text {, measured }}$ is junction temperature obtained from measurement and $T_{J}$, estimated is junction temperature obtained from Cauer

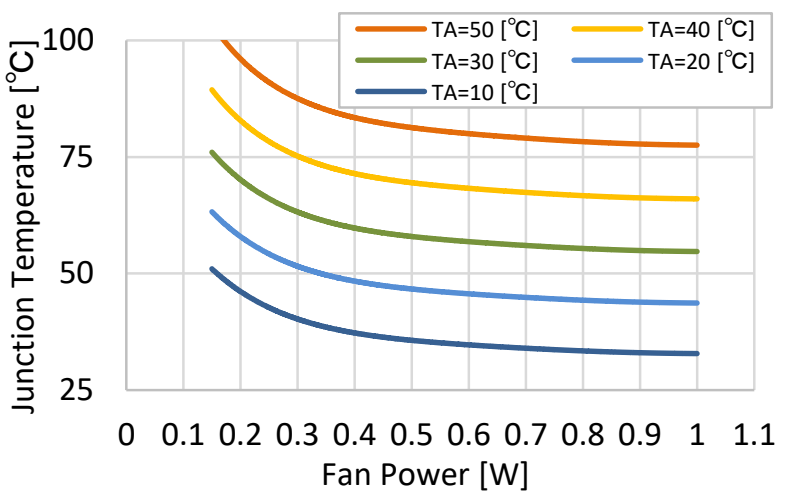

Figure 8. Relationship between microprocessor's junction temperature and fan power.

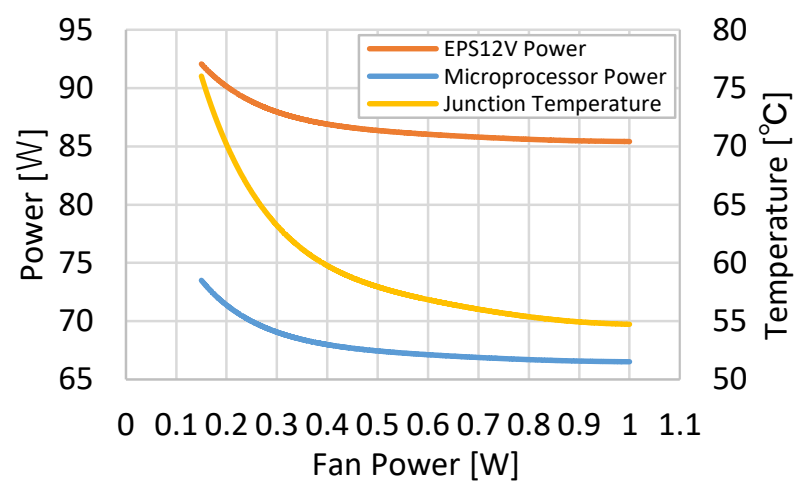

Figure 9. Relationship between microprocessor related power consumption and fan power under $30 \mathrm{deg} \mathrm{C}$ ambient.

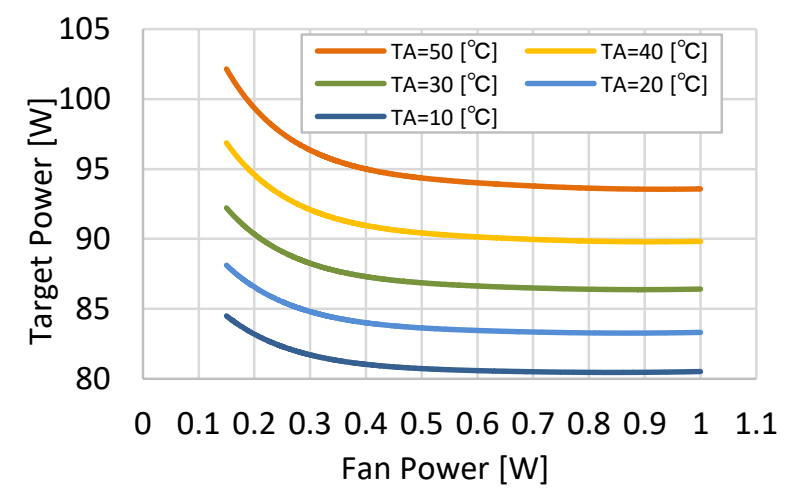

Figure 10. Relationship between sum of "EPS12V power and fan power" and fan power.

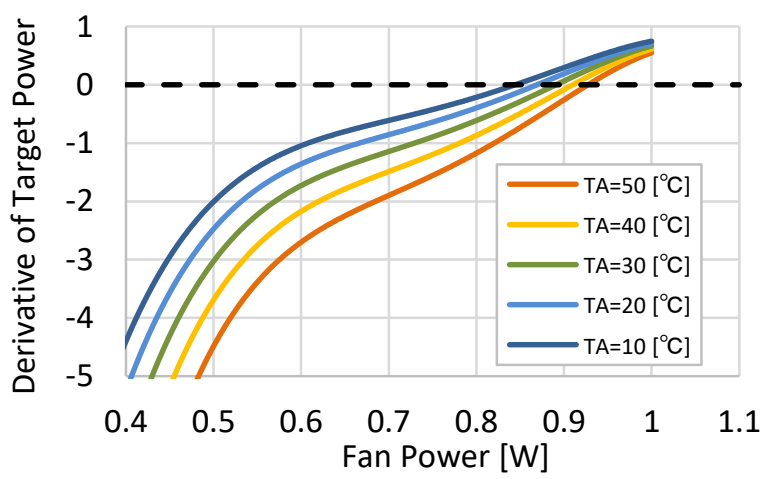

Figure 11. Derivative of sum of "EPS12V power and fan power" curve. 


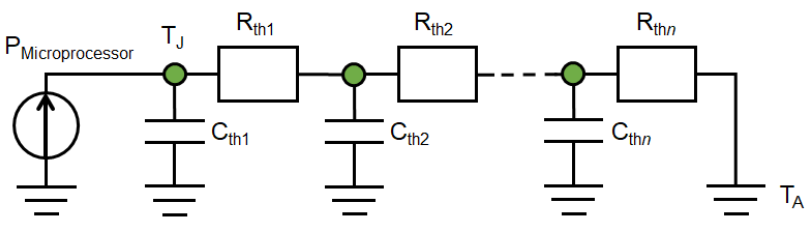

Figure 12. Cauer RC thermal network.

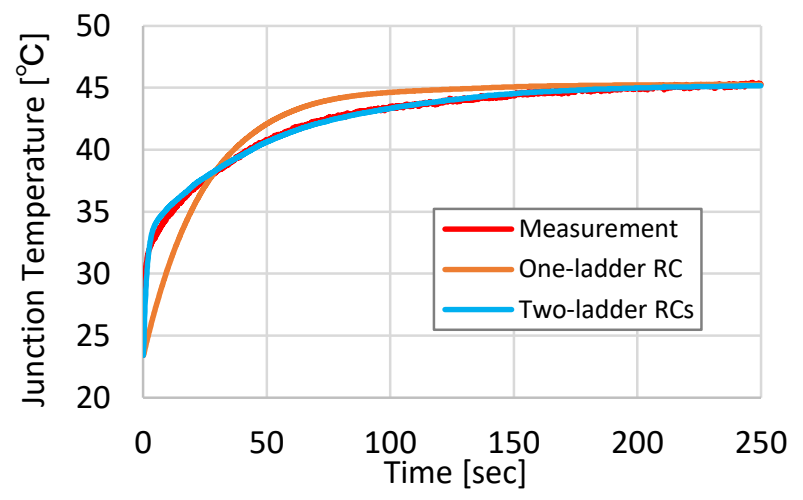

Figure 13. Transient curve of microprocessor junction temperature.

\section{RC thermal network.}

Figure 13 shows fitting results with one-ladder and two-ladder Cauer RC network utilizing OpenModelica. One-ladder RC network shows obvious difference from measurement, while two-ladder RC network fits well compared to one-ladder RC network. By adding more ladders, estimated junction temperature will fit more with measurement. Transient fan control optimization with Cauer RC network will be the future work of this research.

\section{Conclusions}

This paper investigates power optimization of microprocessor dedicated power rail, EPS12V, targeting a desktop computer system under a constant CPUintensive workload condition. Microprocessor and EPS12V power models and heat transfer path model as a variable thermal resistance by fan power are created as Modelica components based on measurement data. By connecting them each other, and optimum fan control condition is explored. It is found that an optimum fan working point exists to minimize sum of EPS12V and fan power. By extending a variable thermal resistance to Cauer RC thermal network, transient heat transfer path is modeled. Transient fan control optimization with Cauer RC network will be the future work of this research.

\section{References}

AMD $\mu$ Prof,

URL https://developer.amd.com/amd-uprof/ (Accessed: 2020-02-10)

AMD Ryzen ${ }^{\mathrm{TM}} 51500 \mathrm{X}$ Processor,

URL https://www.amd.com/en/products/cpu/amd-ryzen-51500x (Accessed: 2020-02-10)

B. Amelifard, F. Fallah and M. Pedram. Leakage minimization of SRAM cells in a dual-Vt and dual-Tox technology, IEEE Trans. on VLSI Systems 16(7): 851-860, July 2008.

L. Codecasa, V. d'Alessandro, A. Magnani, N. Rinaldi, P. J. Zampardi. Fast novel thermal analysis simulation tool for integrated circuits (FANTASTIC), Proceedings of THERMINIC 2014, September 2014. doi: 10.1109/THERMINIC.2014.6972507

A. Gödecke, M. Mühlbauer, J. Nieveler, I. Vittorias, T. Vontz. FEM models in System Simulations using Model Order Reduction and Functional Mockup Interface, Proceedings of the 9th International Modelica Conference: 565-570, September 2012. doi: 10.3384/ecp12076565

T. Hiramoto, K. Uchida, N. Sugii and K. Takeuchi. Integrated Nanodevice, Maruzen (ISBN 978-4-621-08208-9): 131, 143-147, November 2009 (In Japanese).

M. Ishizuka and Y. Fukuoka. Transient Temperature Rise for Multi-Chip Packages, Transactions of the Japan Society of Mechanical Engineers. B 52(476): 1772-1776, April 1986. doi: 10.1299/kikaib.52.1772 (In Japanese)

N. Kunimine, et al. The Newest Thermal Design Technique and Electronics Cooling Technology, CMC Publishing (ISBN 978-4-7813-0510-3): 60-61, December 2011. (In Japanese)

K. Nishi. Transient Heat Conduction Simulation around Microprocessor Die, Thermal Science \& Engineering 20(2):27-34, April 2012. doi: 10.11368/tse.20.27 (In Japanese)

K. Nishi and T. Kaneda. Microprocessor Electrical-Thermal Co-Simulation Model Creation for Low Power Conservation of Computer Systems, OpenCAE Symposium 2019, B-3, December 2019. (In Japanese)

OpenModelica, URL https://www.openmodelica.org (Accessed: 2020-0210)

Prime 95,

URL https://www.mersenne.org/download/\#stresstest (Accessed: 2020-02-10)

K. Uchida, J. Koga, R. Ohba, T. Numata, and S. Takagi. Experimental Evidences of Quantum-Mechanical Effects on Low-field Mobility, Gate-channel Capacitance, and Threshold Voltage of Ultrathin Body SOI MOSFETs, Technical Digest of International Electron Devices Meeting (IEDM): 633-636, December 2001.

K. Uchida, H. Watanabe, A. Kinoshita, J. Koga, T. Numata, and S. Takagi. Experimental Study on Carrier Transport Mechanism in Ultrathin-body SOI n- and p-MOSFETs with SOI Thickness less than $5 \mathrm{~nm}$, Technical Digest of International Electron Devices Meeting (IEDM): 47-50, December 2002. 
H. Watanabe, K. Uchida, and A. Kinoshita. Numerical Study of $\mathrm{C}-\mathrm{V}$ Characteristics of Double-Gate Ultrathin SOI MOSFETs, IEEE Transactions of Electron Devices 54(1): 52-58, January 2007. 